EUROPEAN ORGANIZATION FOR NUCLEAR RESEARCH

CERN-PS/98-047 (CA)

October 5, 1998

\title{
Experimental Area of the CERN Antiproton Decelerator
}

\author{
M. Giovannozzi, J.-Y. Hémery, C. Metzger, U. Mikkelsen ${ }^{1)}$
}

\begin{abstract}
The CERN Antiproton Decelerator will deliver low energy beams to four experimental beam-lines installed within the accelerator circumference. The limited space available imposes tight constraints on the topology of the beam-lines needed by the experimental devices. In this paper, the general layout of the experimental area is reviewed. Furthermore, a discussion of the so-called measurement line for AD beam property measurements is included.
\end{abstract}

Presented at the Biennal Conference on Low Energy Antiproton Physics - LEAP98 Villasimius, Cagliari, Italy

7-12 September 1998

\footnotetext{
1) Institute for Storage Ring Facilities, ISA, University of Aarhus, DK-8000 Aarhus, Denmark
} 


\section{$1 \quad$ Introduction}

Three Collaborations have been officially accepted to take data using the low energy antiproton beams delivered by the CERN AD machine. They are

- ASACUSA - AD-3. In a first stage, this collaboration will pursue atomic physics experiments. In particular, spectroscopy of antiprotonic Helium atoms, but also ionization studies and measurements of energy loss of antiprotons at low energy. At a later stage, it is planned to produce $\mathrm{p} \overline{\mathrm{p}}$ atoms [1].

- ATHENA - AD-1. The collaboration intends to produce antihydrogen, $\overline{\mathrm{H}}$, nearly at rest and in sufficient amounts to be able to perform spectroscopy. The long-term aim is a test of CPT invariance, by a comparison to normal hydrogen with a relative precision of as little as $10^{-15}$ [2].

- ATRAP - AD-2. Also in this case, the aim is to synthesise and study anti-hydrogen in particular to do spectroscopy. The antihydrogen will be produced by exploiting the recombination of $\overline{\mathrm{p}}$ and $\mathrm{e}^{+}$at high $\mathrm{e}^{+}$density.

Furthermore, the ATRAP collaboration will continue the experiments devoted to the precise measurement of the $\bar{p}$ mass [3].

In order to satisfy the requests of the three Collaborations, four experimental transfer lines have been designed: two will be used by ATRAP. ASACUSA and ATHENA will have one beam line each. A fifth general purpose transfer line is foreseen for measurements of some parameters related to the AD machine and beam.

All the experimental lines will be installed inside the machine circumference, thus imposing tight constraints on the design of the experimental areas due to the limited space available. Therefore, the beam lines have to be kept as short as possible and their geometry has to be carefully chosen in order to increase the distance between experimental lines, thus reducing the electro-magnetic influence between neighbouring lines.

\section{$2 \quad$ Experimental beam lines}

A section of the AD machine is shown in Fig. 1 together with the schematic layout of the experimental beam lines. The experimental lines are connected to the AD machine via the 7000, DE0 lines. Three of them are horizontal beam lines (DE1, DE2, DEM), while two are vertical (DE3, DE4).

The optics of the experimental lines has been designed in order to have a minimum beam size and zero dispersion function at the focus location. The computations of the optics parameters have been performed by using the MAD program [4]. The beam parameters used to compute the evolution of the beam envelope are $\varepsilon_{H}=\varepsilon_{V}=5 \pi \mathrm{mm} \cdot \mathrm{mrad}$ and $\Delta p / p=1 \times 10^{-3}$. All the values refer to $95 \%$ of the distribution and are conservatives estimates.

The beam emittance is expected to be larger than the nominal value quoted in [5], with a minimum value of $\simeq 1.5 \pi \mathrm{mm} \cdot \mathrm{mrad}$, owing to rest-gas scattering at the final momentum of $100 \mathrm{MeV} / \mathrm{c}$. Furthermore, due to variations of the elements in the AD and its transport lines between different extractions, the area in phase-space covered by the beam over several shots will be larger than the true emittance. This so-called overall effective emittance is expected to be up to $\approx 10 \pi \mathrm{mm} \cdot \mathrm{mrad}$.

The optics of the DE0 line has been computed to match the optical parameters in the different experimental lines. These optics can be labelled according to the physics program of the ASACUSA collaboration which will determine their use: 
- Phase 1: during this phase the ASACUSA Collaboration will use a thin degrader to slow down the antiprotons delivered by the AD machine;

- Phase $2 \& 3$ : in this phase the reduction of the kinetic energy of the antiproton beam will be performed by using a Radio Frequency Quadrupole (RFQ) (see Ref. [6] for more details on this part of the project). This device will be installed in the DE1 line after the completion of the data-taking foreseen for Phase 1;

- Phase (2 \& 3)': after the installation of the RFQ, it is envisaged to pursue some additional studies using a thin degrader (hence resuming a Phase 1-like data-taking). These studies will be carried out during the stops imposed by technical reasons to the normal Phase $2 \& 3$ program;

A detailed description of the optics studied can be found in $[7,8,9,10]$.

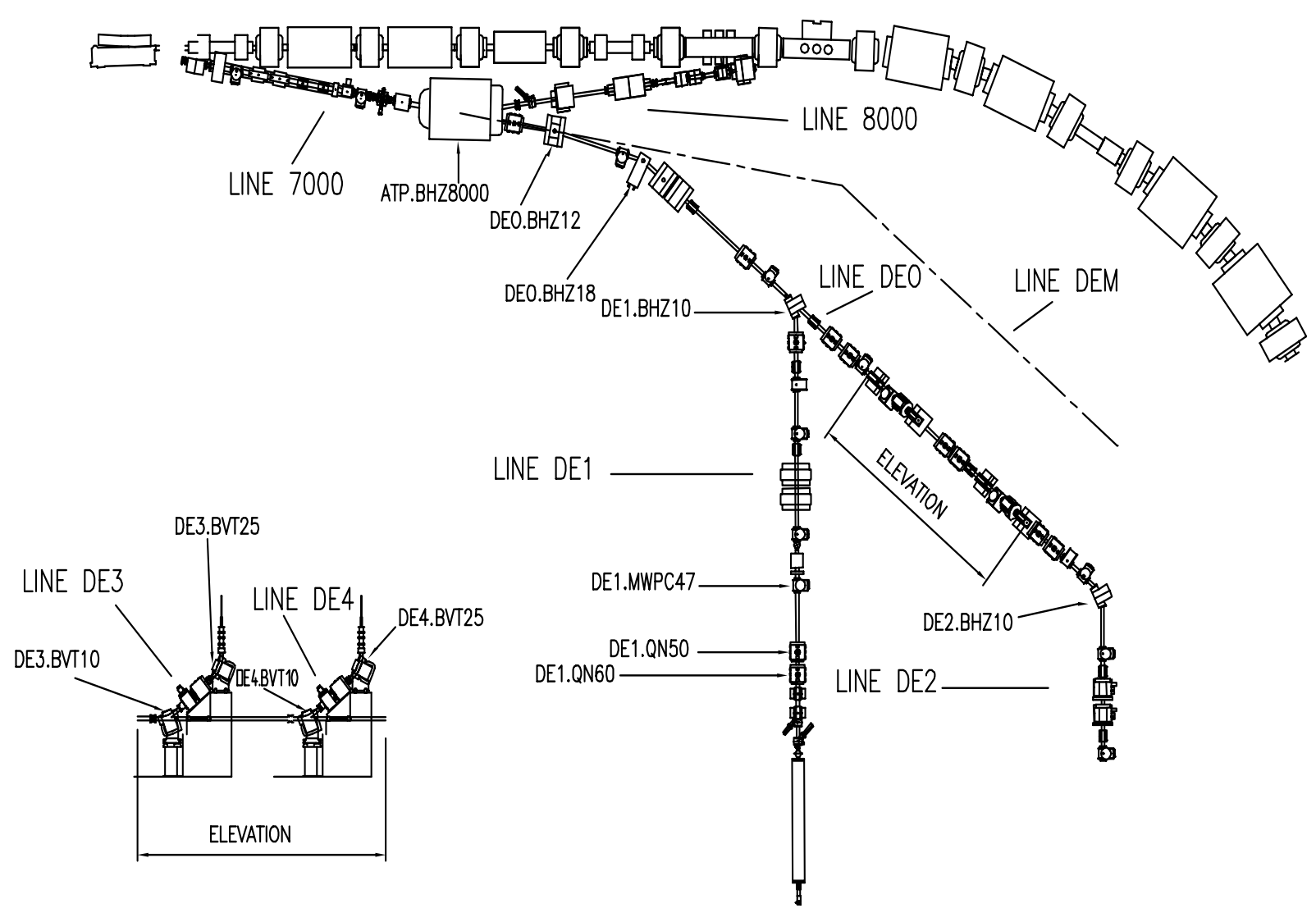

Figure 1: The overall view of the $\mathrm{AD}$ beam lines. The four experimental beam lines DE1, DE2, DE3, DE4 are shown, together with DE0 and the measurement line DEM. The $7000 / 8000$ transfer lines are also visible.

\section{$2.1 \quad$ Line DEO}

The DEO line is the main branch of the whole set of transfer lines. It is connected to the extraction line 7000 through the bending magnet ATP.BHZ8000, which acts as a drift space in the case of antiproton extraction at $100 \mathrm{MeV} / \mathrm{c}$. An overall bending angle of $30^{\circ}$ is performed using two horizontal C-shaped bending magnets. The first magnet (DE0.BHZ12) deflects the beam by $7^{\circ}$, while the second one (DE0.BHZ18) increases the 
bending angle by $23^{\circ}$. The first dipole acts as a switching magnet to deliver beam to the ensemble of experimental lines DE1, DE2, DE3, DE4, when the bending angle is set to $7^{\circ}$, or to the measurement line DEM, in case the bending angle is zero. Downstream of the second dipole, the DE0 transfer line is simply a straight section.

Four bending magnets allow to deliver the beam to the four experimental zones. The deflection angle is of $50.4^{\circ}$ (in the horizontal plane) for DE1, DE2 and $39.6^{\circ}$ (in the vertical plane) for DE3, DE4. With such a choice for the geometry of DE0, the DE1 and DE2 lines are roughly perpendicular to the major axis of the AD machine. The axis of the horizontal beam lines is located at $1.2 \mathrm{~m}$ above the ground level.

Due to severe constraints for the field stability of these magnets - which will affect the so-called overall effective emittance - the switching time will be of the order of 5 minutes and thus pulse-to-pulse switching of the beam among the experiments cannot be performed.

The outcome of the optics computations is the following:

- Phase 1: during this phase, the part of the DE0 upstream of the bending magnet DE1.BHN10, will have two different settings: one used to deliver the beam to the DE1 line and a second one to match the beam in the other lines DE2, DE3, DE4. More details can be found in $[7,8]$.

- Phase 2 \& 3: for this configuration the same two optics designed for Phase 1 will be used.

- Phase (2 \& 3)': for this configuration only one optics will be used to deliver beam to each of the four experimental lines.

\section{$2.2 \quad$ Line DE1}

This line starts with the horizontal bending magnet DE1.BHN10. Five quadrupoles are installed along the line, while the trajectory correction is performed using up to four combined function (horizontal/vertical) steering magnets. Three wire chambers are installed in the line to measure the beam properties (position and width).

This line will be used in different modes, according to the experimental set-up used for the data-taking. Two different optics have been computed to achieve the necessary beam quality:

- Phase 1: the optics used is presented in Fig. 2. In the upper part are shown the beam envelopes - horizontal and vertical - for this line. It is used to deliver the beam to ASACUSA, starting at the AD ejection septum, through the 7000 line, the DEO line and finally the DE1 line.

- Phase $2 \& 3$ : the optics is the same as for Phase 1. The entrance of the RFQ is located $0.22 \mathrm{~m}$ upstream of the focusing point used in Phase 1;

- Phase (2 \& 3)': the physics run using the RFQ to decelerate the antiproton beam represents a challenge both from the point of view of the transfer line and the RFQ performance (see Ref. [6] for more details). To resume a Phase 1-like run, the solution proposed consists in moving further upstream the focusing point, almost at the location of the monitor DE1.MWPC45: the section of beam line between such a monitor and the quadrupole DE1.QN60 will be dismantled (removing the buncher and the wire chamber DE1.MWPC47) to leave some space for the experimental apparatus. Thus, the critical elements, quadrupoles and the RFQ, will remain in place. The optics is shown in Fig. 2 (lower part). 


\section{$2.3 \quad$ Line DE2}

The DE2 line delivers beam to the ATHENA experimental area. The bending magnet DE2.BHZ10 deflects the antiprotons by an angle of 50.4 . Two quadrupole magnets allow to focus the beam $1.5 \mathrm{~m}$ downstream of the doublet. Two combined function steering dipoles (horizontal/vertical) are foreseen to compensate the residual trajectory errors measured by means of two Multi Wire Proportional Chambers (MWPCs). In order to facilitate the commissioning of the beam line, it is also foreseen to install a MWPC at the focus location.

\subsection{Lines DE3 and DE4}

The DE3, DE4 lines deliver the beam to the ATRAP experimental apparatus. They look very similar, as far as the geometry and the magnetic elements are concerned. The overall design has been inherited from the layout of the experimental beam line used by the PS196 Collaboration installed in the South Hall [11] of the LEAR machine.

The main feature is that they are vertical lines: the beam is bent vertically by $90^{\circ}$ in two steps, $39.6^{\circ}$ and $50.4^{\circ}$, by means of two dipoles. The transfer lines are rather short, their lengths being $4 \mathrm{~m}$. This imposes tight constraints in the design of the optics. Only one quadrupole can be installed in each line and this is neither enough to control the transverse beam parameters nor the horizontal and vertical dispersion function. Hence, it is mandatory to carefully choose the optical parameters at the entrance of the lines, in order to obtain a reasonable beam size at the focus located at an altitude of $4.44 \mathrm{~m}$ above the ground level. An extra parameter that can be used to optimise the optics is the additional focusing force due to the edge effect of the bending magnets. The entrance and exit angles can be chosen in such a way that the net effect is equivalent to a thin quadrupolar lens. This is obtained by adding polar pieces at the magnet ends with the proper angle. The optimal angles are the same as those used in the South Hall, namely a single polar piece at the magnet exit having an angle of $10.4^{\circ}$.

An additional constraint in the design of the vertical lines, is the need for a port below the last vertical bending magnet to observe the antiproton beam passing through. Furthermore, a minimum space of $0.40 \times 0.40 \mathrm{~m}^{2}$ has to be reserved on the ground for the device used to observe the beam. A solution could be found, so that the quadrupoles in the DEO line do not interfere with such a special requirement. As far as the vacuum parts are concerned, it is necessary to build special Y-shaped chambers for the vertical bending magnets and the horizontal vacuum pipe should be replaced by X-shaped sections to allow the beam to pass through the horizontal beam line while a vacuum pipe connects the port below the last vertical bending magnet of the DE3, DE4 lines to the device used to look at the beam.

The reduction of the horizontal beam trajectory in DE3 and DE4, can be performed by using the dedicated correctors in the DEO line, while the vertical trajectory is compensated by means of the two vertical bending magnets used also to reduce steering errors upstream of the two vertical lines. The beam trajectory is measured by a multi wire proportional chamber and a second monitor, provided by the Collaboration, placed at the focus location.

In Table 1 are reported the horizontal and vertical beam size together with the horizontal and vertical beam divergence at the focus location for the four experimental beam lines. 


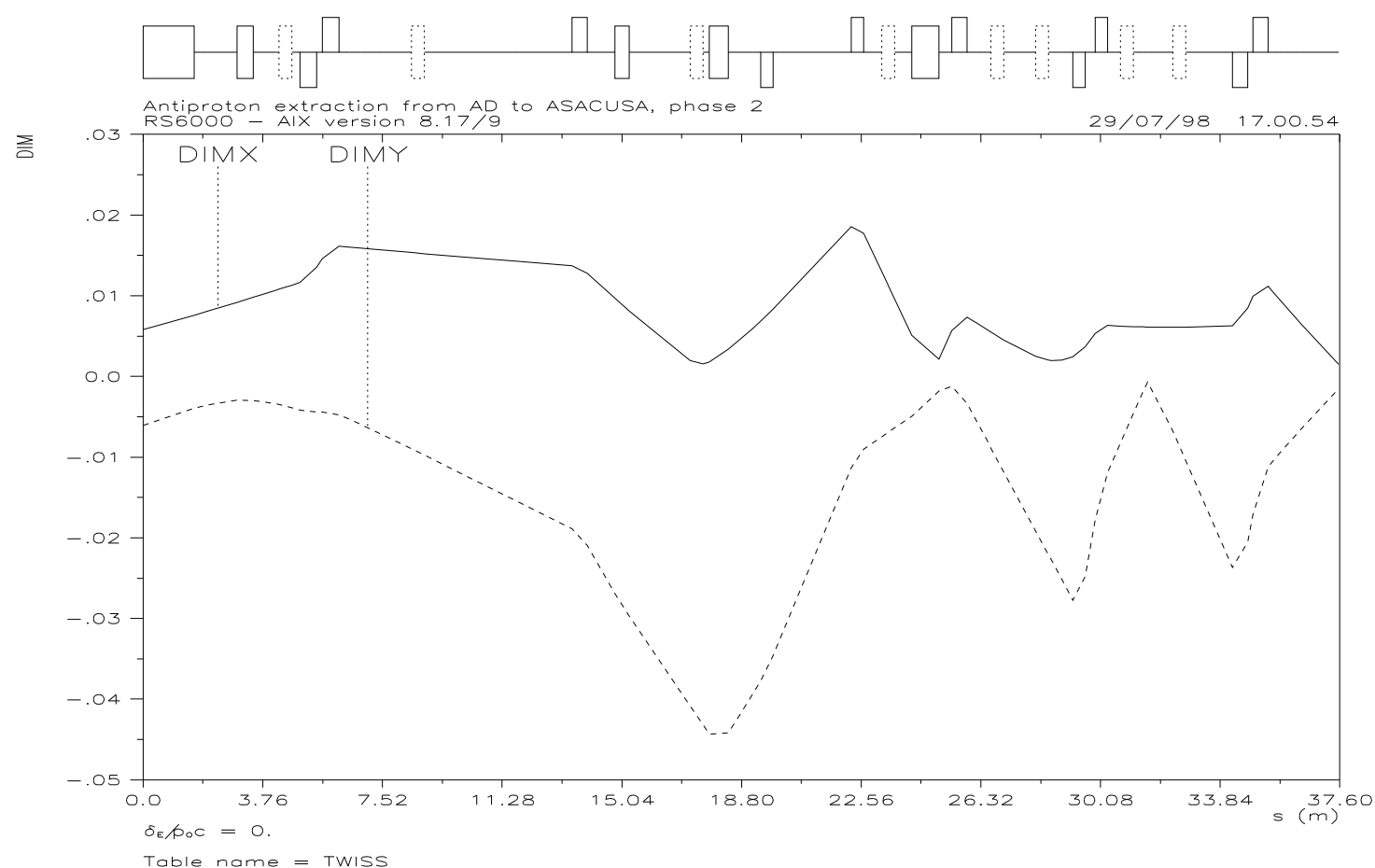

Table name $=$ Twiss

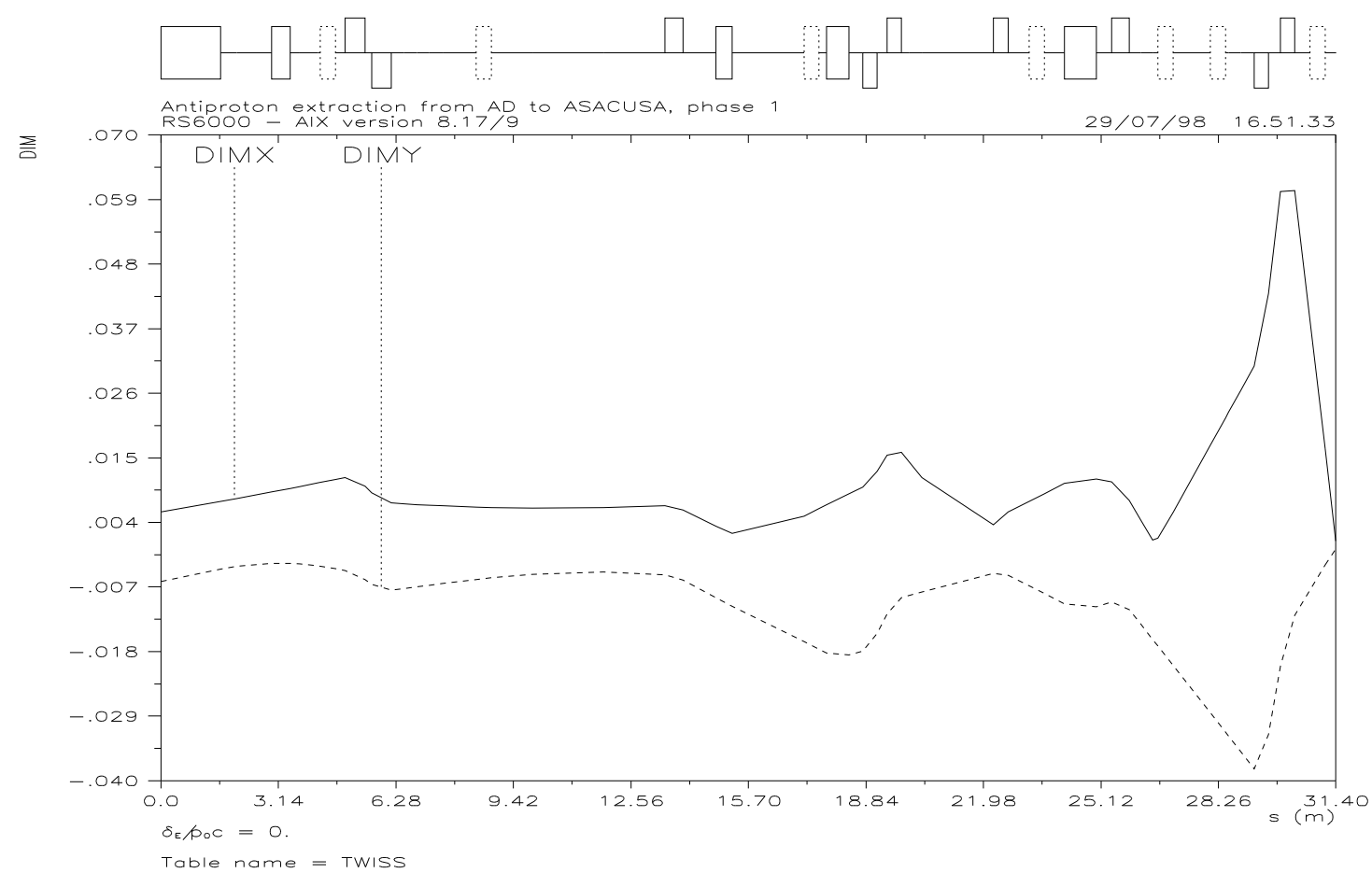

Figure 2: Horizontal and vertical beam envelope for the DE1 (ASACUSA) line, starting from the AD ejection septum. The beam envelope refers to $95 \%$ of the beam. This optics is the one used in Phase 1 and Phase (2 \& 3) (upper curve) and Phase (2 \& 3)' (lower curve). 


\begin{tabular}{|c|c|c|c|c|c|c|}
\hline & \multicolumn{6}{|c|}{ Beam parameters } \\
\hline & \multicolumn{2}{|c|}{ Phase 1} & \multicolumn{2}{|c|}{ Phase $2 \& 3$} & \multicolumn{2}{|c|}{ Phase (2\& 3)' } \\
\hline & $\begin{array}{c}\text { Size }(\mathrm{H} / \mathrm{V}) \\
{[\mathrm{mm}]}\end{array}$ & $\begin{array}{c}\text { Div. }(\mathrm{H} / \mathrm{V}) \\
{[\mathrm{mrad}]}\end{array}$ & $\begin{array}{c}\text { Size }(\mathrm{H} / \mathrm{V}) \\
{[\mathrm{mm}]}\end{array}$ & $\begin{array}{c}\text { Div. }(\mathrm{H} / \mathrm{V}) \\
{[\mathrm{mrad}]}\end{array}$ & $\begin{array}{c}\text { Size }(\mathrm{H} / \mathrm{V}) \\
{[\mathrm{mm}]}\end{array}$ & $\begin{array}{c}\text { Div. }(\mathrm{H} / \mathrm{V}) \\
{[\mathrm{mrad}]}\end{array}$ \\
\hline$\overline{\mathrm{DE} 1}$ & "1.10/1.10 & $\overline{4} 4.56 / 4.56$ & $-1-$ & 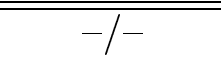 & (0.20/0.53 & $4 \quad 48.55 / 9.46$ \\
\hline DE2 & $0.24 / 0.38$ & $30.68 / 13.23$ & $0.24 / 0.38$ & $30.68 / 13.23$ & $0.24 / 0.38$ & $30.68 / 13.23$ \\
\hline DE3 & $1.77 / 1.62$ & $3.34 / 4.25$ & $1.77 / 1.62$ & $3.34 / 4.25$ & $1.77 / 1.62$ & $3.34 / 4.25$ \\
\hline $\mathrm{DE} 4$ & $0.61 / 1.41$ & $8.92 / 3.57$ & $0.61 / 1.41$ & $8.92 / 3.57$ & $0.61 / 1.41$ & $8.92 / 3.57$ \\
\hline
\end{tabular}

Table 1: Horizontal and vertical beam size and divergence at the focus location for the four experimental beam lines. The values refer to $95 \%$ of the beam. The beam size and divergence for the DE1 (ASACUSA) line during Phase $2 \& 3$ is not shown, as there is no focal point foreseen for the RFQ optics.

\section{Measurement line}

The so-called measurement line, DEM, is used for two main purposes [9]:

- Beam property measurements: the transverse emittance and the momentum spread are vital parameters for the AD machine. The transverse emittance is proposed to be measured by a variation of the standard method [12] where the beam size is detected in three MWPCs. Due to the space restrictions, this method has been limited to the use of two MWPCs, where the symmetry then must be assured by adjustment of the focal length of the quadrupole to coincide with the distance between this and the second MWPC. In this way, the emittance is determined simply from the ratio of the widths of the beam observed in the two MWPCs when the dispersion is set to zero.

The momentum spread is measured by increasing the dispersion invariant after the $30^{\circ}$ deflection before the focus. The optics has been designed such that upstream the $30^{\circ}$ deflection, the dispersion is essentially zero in the MWPC while the beam size caused by the transverse emittance is large, whereas downstream the bend the dispersion is large and the $\beta$-function small. This allows a determination of $\Delta p / p$.

- RFQ matching: Since the deceleration of antiprotons with a RFQ is technically demanding, it is necessary to be able to test the operation before installation in the DE1 line. Optics requirements similar to those for the ASACUSA line have been fulfilled thus allowing to perform the tests on this beam line.

\section{$4 \quad$ Safety issues and layouts}

A thorough study has been carried out in order to make the experimental area complying with the Safety Rules in force at CERN. We will only mention here some aspects which are strictly related with the design of the experimental areas:

- Personnel evacuation: the former AA/AC building, the actual AD Hall, was not conceived to be used as an experimental area where people are supposed to stay during the functioning of the machine. The scenario will be completely different during the physics run of the $\mathrm{AD}$ machine: about forty physicists will stay in the area during the data-taking. For this reason, the installations have been designed to allow an easy access/evacuation of the area.

A new access door, has been foreseen in addition to the old door installed in the $D$ quadrant of the building 193 . The new door will be placed in the $B$ quadrant 
to allow a second emergency escape path. All the installations, i.e. experimental areas, counting rooms, laser huts and workshops, can be accessed/evacuated by using at least two different paths. The details of this structure can be seen in Fig. 3, where the overall layout of the experimental zones, including the infrastructures, is reported. In Fig. 4 a 3D view of the experimental area is shown.

- Radiation protection: due to the capability of the AD machine to work with both protons and antiprotons, the experimental Hall will be considered differently as far as the access is concerned. No access to the experimental area will be possible during the operation of the machine with protons. On the other hand almost no restriction will be imposed on the access to the Hall during the standard operation with antiprotons. Furthermore, due to the rather low intensities (about $10^{7} \overline{\mathrm{p}}$ in non-stacking mode) delivered by the AD machine during the antiproton runs and the low energy of the particles transported through the beam lines, the concrete walls separating the different experimental areas are $0.40 \mathrm{~m}$ thick to allow a better use of the limited space available.

\subsection{ASACUSA Collaboration}

The ASACUSA experimental area occupies a surface in the $B, C$ and $D$ quadrants. It allows the installation of the complex detectors and of the RFQ used in Phase $2 \& 3$. The access door is installed at the back of the area. The counting rooms are installed along the major side of the area. Two $4 \times 12 \mathrm{~m}^{2}$ barracks are mounted on a metallic structure $3 \mathrm{~m}$ high. Both the barracks and the structure will be recuperated from the South Hall stock. Almost at the centre of the experimental Hall is located the special laser hut, an $8 \times 8 \mathrm{~m}^{2}$ structure where all the equipment related with the lasers to be used during the data-taking is installed and operated.

\subsection{ATHENA Collaboration}

The ATHENA experimental area is almost entirely located in the $C$ quadrant. It has a slightly smaller surface then the ASACUSA zone. The access through the standard door is on the back of the area.

The counting room, a $4 \times 12 \mathrm{~m}^{2}$ barrack, is installed on a metallic structure $3 \mathrm{~m}$ high, located between the experimental area and the machine circumference (see drawing on page 8 ). The laser hut, likewise a $4 \times 12 \mathrm{~m}^{2}$ structure, is placed underneath.

\subsection{ATRAP Collaboration}

The design of the ATRAP experimental area represents one of the most challenging aspects of the whole AD experimental complex.

The use of vertical beam lines imposes to install the experimental apparatus on a platform located at $3 \mathrm{~m}$ from the floor. Although the details concerning the construction of such a platform are not known yet, the assumptions are to build a concrete structure $0.20 \mathrm{~m}$ thick.

Two independent areas are delimited by building $0.60 \mathrm{~m}$ thick concrete walls around the vertical beam pipes. The increase in the wall thickness allows to shield the sensitive part of the apparatus from the background noise induced by the strong radioactive sources needed during some phases of the data-taking. The details of the platform can be found in Fig. 5.

The counting room, $4 \times 12 \mathrm{~m}^{2}$, is installed on the same metallic structure used to support the ASACUSA counting rooms. An easily removable walkway, located on the DEM 


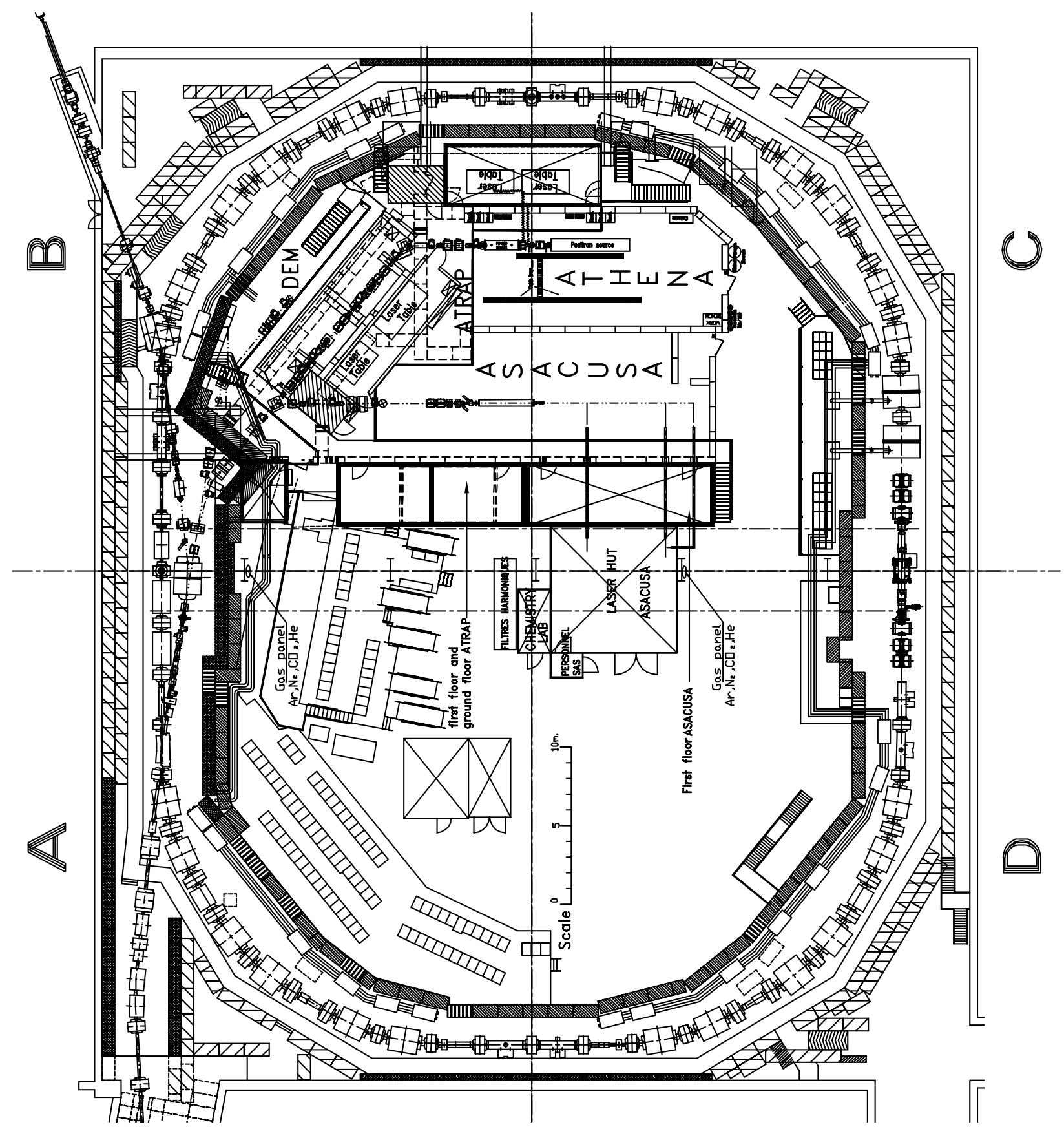

Figure 3: 2D view of the AD Experimental Area.

side of the ATRAP platform, connects the counting room to both zones allowing to transport small parts without using the crane. In addition, a $4 \times 6 \mathrm{~m}^{2}$ barrack is installed on the ground, underneath the ATRAP counting room, to serve as a workshop.

\subsection{DEM area}

The schematic layout of the DEM zone has been studied. The access door is placed on the ATRAP platform, near the Faraday Cage (see Fig. 5). Special care has been taken to leave an easy access to the zone with the crane, this in order to facilitate the installation 


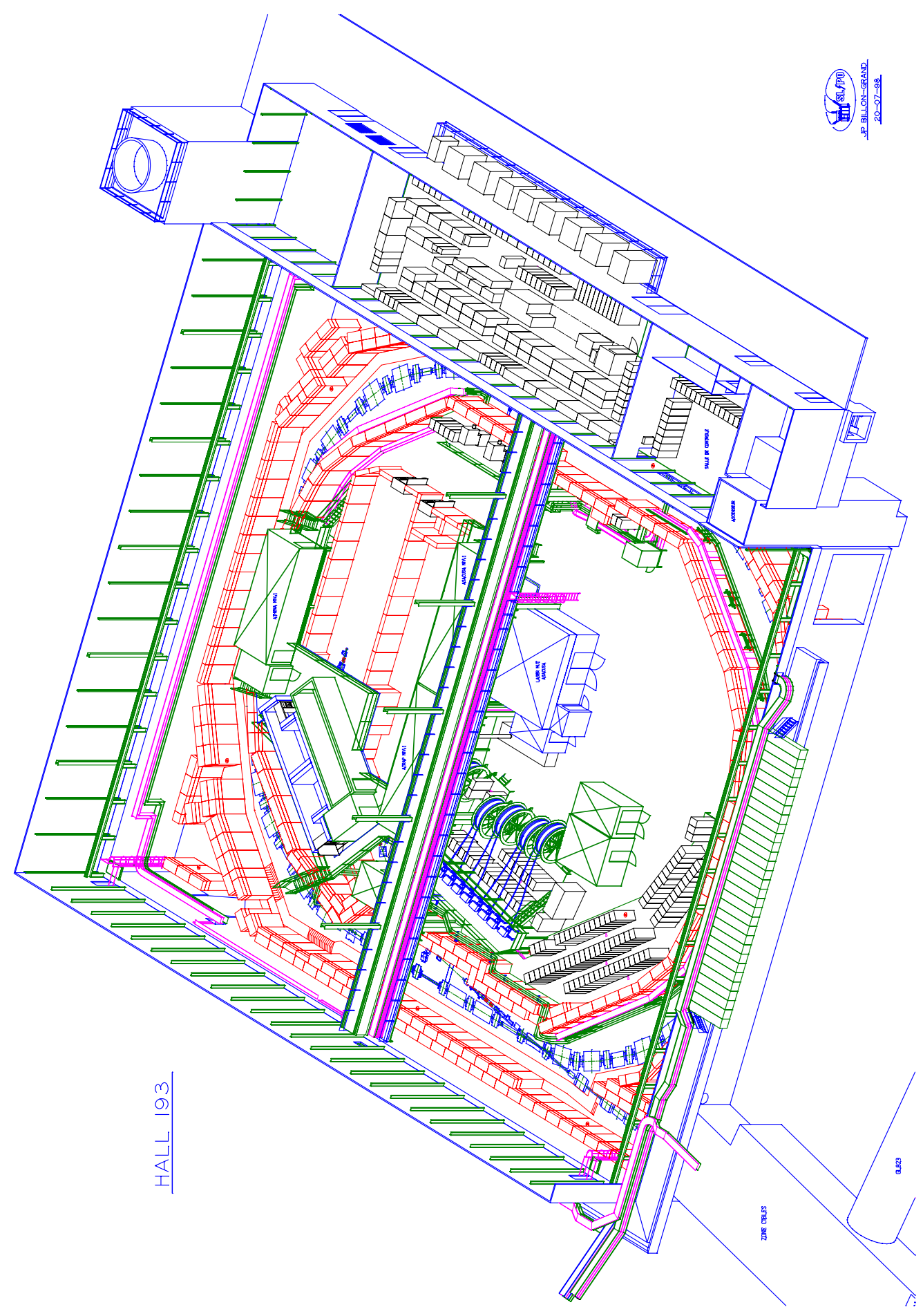

Figure 4: 3D view of the AD Experimental Area.

of the magnetic elements of the transport line, and the experimental apparatus.

The counting room is a standard $4 \times 3 \mathrm{~m}^{2}$ barrack to be installed near the injection/extraction region of the $\mathrm{AD}$ machine.

\section{Acknowledgements}

We would like to thank the other members of the AD team for many stimulating discussions and constructive criticism. 


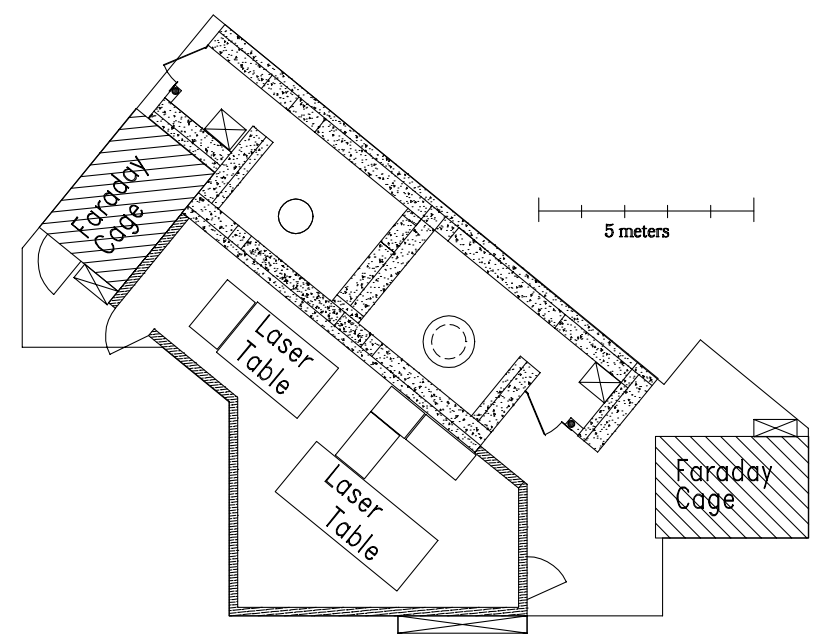

Figure 5: layout of the ATRAP platform.

One of the authors (U.M.) acknowledges support from the Danish Natural Science Research Council under grant No. 9701361.

\section{References}

[1] ASACUSA Collaboration, (1997) "Atomic spectroscopy and collisions using slow antiprotons", CERN SPSC 97-19.

[2] ATHENA Collaboration, (1996) "Antihydrogen Production and Precision Experiments", CERN SPSLC 96-47.

[3] ATRAP Collaboration, (1997) "The production and study of cold antihydrogen", CERN SPSC 97-8.

[4] H. Grote, F. C. Iselin, (1990) "The MAD Program, User's Reference Manual", CERN SL (AP) 90-13.

[5] S. Baird et al., (1996) "Design study of the Antiproton Decelerator: AD", CERN PS (AR) 96-43.

[6] J. Bosser et al., (1997) "Feasibility study of a decelerating Radio Frequency Quadrupole for the Antiproton Decelerator AD", CERN PS/Note (HP) 97-36.

[7] U. Mikkelsen, (1998) "Injection of protons and extraction of antiprotons at the Antiproton Decelerator", CERN PS Note (CA) 98-08.

[8] M. Giovannozzi, (1998) "Experimental transfer lines and areas for the AD machine", CERN PS Note (CA) 98-13.

[9] U. Mikkelsen, (1998) "The measurement line at the Antiproton Decelerator", CERN PS Note (CA) 98-19.

[10] C. Metzger, (1997) "AD Project. Optics calculations for the transfer lines. First attempt", CERN PS Note (CA) 97-17.

[11] J-Y. Hémery and D. J. Simon, (1991) "The LEAR Experimental Areas, Present layout", CERN PS (PA) 91-13.

[12] M. Arruat and M. Martini, (1992) "The new standard method to measure emittance in the PS transfer lines", CERN PS (PA) 92-59.

[13] S. P. Møller et al., (1997) "Direct measurements of the stopping power for antiprotons of light and heavy targets", Phys. Rev. A 56, 2930. 